\title{
Survival prognostic factors and markers of morbidity in Spanish patients with systemic sclerosis
}

Carmen-Pilar Simeon, Luis Armadans, Vicent Fonollosa, Miquel Vilardell, Jaume Candell, Carles Tolosa, Fermín Mearin, María José Rodrigo, Roser Solans, Joan Lima, Gabriel Sampol

Department of Internal Medicine C-P Simeon V Fonollosa M Vilardell C Tolosa

R Solans

J Lima

Service of Preventive Medicine and Epidemiology

L Armadans

Service of Cardiology J Candell

Service of Gastroenterology F Mearin

Department of Biochemistry and Immunology M J Rodrigo

Service of Pneumology G Sampol

Hospital General Universitari Vall D'Hebron, Barcelona, Spain

Correspondence to: Dr C P Simeon, C/ Provenza 473 sat $2^{\mathrm{a}}$, 08025 Barcelona, Spain.

Accepted for publication 10 September 1997

\begin{abstract}
Objective-To identify survival prognostic factors and markers of morbidity among patients with systemic sclerosis (SSc). Patients and Methods-The study included 72 patients diagnosed with SSc. According to the extent of skin involvement, three groups of patients were established: group 1, without sclerosis and with sclerosis of fingers and neck; group 2, with sclerosis of face and distal to elbows and knees; group 3 , with generalised sclerosis including the trunk. All patients were included in a study protocol to determine visceral involvement. Cumulative survival after first symptom has been estimated according to the Kaplan-Meier method. The association between a hypothetical prognostic factor and cumulative survival after first symptom was assessed by $\log$ rank test. The association between a hypothetical risk factor and the prevalence of severe morbity was assessed by the odds ratio. Multiple logistic regression models were used to identify the main predictors of severe morbidity.
\end{abstract}

Results-Survival was estimated to be $85 \% 10$ years after first SSc symptom. Survival was higher among SSc patients with skin involvement distal to elbows and knees than among the rest of patients; a forced vital capacity (FVC) on spirometry lower than $70 \%$ of expected value was associated with a shorter survival, even after adjustment for diffuse SSc. Skin involvement proximal to elbows or knees was associated with a higher prevalence of severe morbidity $(O R=46.57 ; p<0.001)$. According to a multiple logistic regression, severe morbidity was higher among patients with skin involvement proximal to knees or elbows $(\mathrm{OR}=40.92 ; \mathrm{p}<0.001)$ or among patients with pulmonary hypertension detected by Doppler echocardiography $(O R=23.66 \mathrm{p}<0.001)$.

Conclusions-In patients with SSc the extent of skin sclerosis was found to be a determining factor on the prognosis. According to skin sclerosis extent two main subsets of SSc patients with different survival incidence and degree of morbidity could be clearly established: limited SSc, formed by patients with no skin sclerosis or with sclerosis distal to elbows and knees and diffuse SSc, formed by patients with skin sclerosis distal and proximal to elbows and knees. Moreover, lung involvement (FVC $<70 \%$ on survival study and pulmonary hypertension on morbidity study) was an important and independent prognostic factor.

(Ann Rheum Dis 1997;56:723-728)

Systemic sclerosis (SSc), a generalised disease characterised by excessive accumulation of connective tissue components and structural vascular abnormalities has a variable natural history. Although most patients have long survival others progress rapidly to death. Thus, within the term SSc, a wide range of patients with different clinical and prognostic features are included.

The magnitude of cutaneous sclerosis is the essential marker of the SSc. However, the groups established by other authors differed both in number and skin sclerosis extent. Barnett et $a l^{1}$ and Giordano et al ${ }^{2}$ subdivided SSc patients in three skin groups: Barnett's type 1 or Giordano's limited form defined patients with sclerodactyly and "neck sign"; type 2 or intermediate form defined patients with sclerosis proximal to metacarpophalangic joints and distal to shoulders and hips; and type 3 or diffuse form was applied to patients with generalised cutaneous sclerosis. Barnett et $a l^{1}$ and Giordano et $a l^{2}$ also reported the immunological features and survival incidence of their three groups. Whereas the first and last group of these two classifications were similar in both survival incidence and immunology, the middle group of both classifications had different characteristics. Thus, survival incidence and serological profile of Barnett's type 2 patients were similar to that seen on his type 1 and Giordano's intermediate form shared the immunological findings and survival incidence of his diffuse form. Medsger et $a l^{{ }^{4}}$ and later LeRoy et $a \bar{P}^{6}$ advocated a two subtype classification: diffuse SSc for patients with skin involvement proximal to elbows and knees and limited SSc for patients with skin sclerosis distal to elbows and knees, including the face.

Moreover visceral involvement and immunological findings have been considered prognostic factors by some authors and thus kidney and heart involvement have been associated with poor prognosis. ${ }^{7-9}$ In the past few years 
Table 1 Clinical and biological features of 72 patients with $S S c$

\begin{tabular}{ll}
\hline & Number (\%) \\
\hline Women:men & $65(90) 7(10)$ \\
Median age (y) & 54 (range:8-74) \\
Raynaud phenomenon & $72(100)$ \\
Capillaroscopic alterations & $61(84)$ \\
Skin involvement & $64(90)$ \\
Group 1 & $24(33)$ \\
Group 2 & $32(44)$ \\
Group 3 & $16(23)$ \\
Muscle involvement & $61(84)$ \\
Digestive involvement & $63(87)$ \\
Lung involvement & $54(75)$ \\
Cardiac involvement & $62(86)$ \\
Renal involvement & $7(9)$ \\
ANA & $70(97)$ \\
Speckled & $50(69)$ \\
Homogeneous & $15(20)$ \\
Nucleolar & $7(9)$ \\
ACA & $30(41)$ \\
Topoisomerase I & $8(11)$ \\
\hline
\end{tabular}

*Skin groups: group 1 without sclerosis and with sclerosis of fingers and neck; group 2, sclerosis of face and distal to elbows and knees; group 3, generalised sclerosis. ANA: antinuclear antibodies. ACA: anticentromere antibodies.

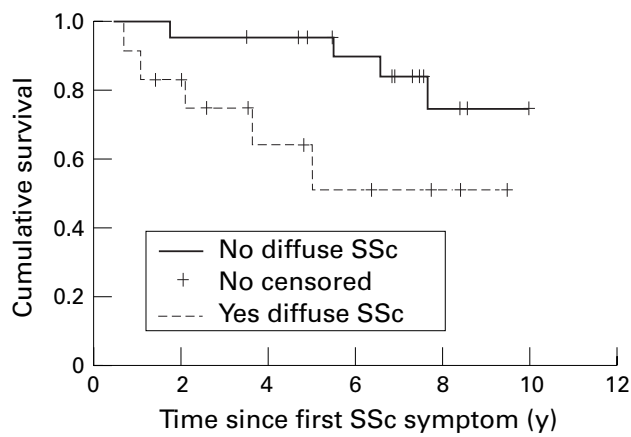

Figure 1 Survival according to extent of skin sclerosis.

some other studies ${ }^{10-14}$ have been conducted to define prognostic factors using multivariate statistical methods but limited conclusions were reached because not all types of SSc patients were included in the studies. Therefore, defining the prognostic factors of SSc is under permanent review because despite an important number of studies, no unanimous conclusions have been drawn. The aim of this study was to analyse and identify survival prognostic factors and markers of morbidity of SSc patients.

\section{Methods}

PATIENTS

The study included 72 patients diagnosed with SSc at the Internal Medicine Department, Hospital General Universitari Vall d'Hebron, from 1980 to 1990 . Fifty nine of 72 patients (81\%) fulfilled the American Rheumatology College (ARC) classification criteria ${ }^{15}$ and 13 patients who did not, had Raynaud's phenomenon, anticentromere antibody (ACA), capillaroscopic abnormalities, sclerodactyly and characteristic visceral involvement of SSc. A detailed protocol containing 150 items of information was completed on each patient during the following 12 months after the diagnosis.
CLINICAL FINDINGS

Skin involvement

Skin sclerosis was considered present when cutaneous thickening was found. The skin sclerosis extent was assessed at diagnosis and six months later by inspection and palpation of the whole body skin by two physicians. Based on their maximum skin sclerosis extent three different groups of SSc patients were established: group 1, with no skin sclerosis or with sclerosis of the fingers and neck; group 2, with sclerosis of face and distal to elbows and knees; group 3, with generalised sclerosis.

Muscle involvement

This was assessed by clinical symptoms (proximal muscle weakness), physical examination, and muscle enzyme determination. If some enzymatic change was found, electromyographic study and muscle biopsy were performed.

foint involvement

This was considered present when arthralgia or arthritis, or tendon friction rubs or acroosteolysis were observed.

Digestive involvement

Digestive involvement secondary to SSc was defined as hypomotility of the lower two thirds of the oesophagus or decreased peristalsis confirmed by manometry or cineradiographic study, or all three.

\section{Pulmonary involvement}

This was considered present when bibasal pulmonary fibrosis on chest radiography or pulmonary functional testing (PFT) alteration (restrictive, obstructive or mixed pattern) on spirometry or less than $70 \%$ of predicted carbon monoxide diffusing capacity according to the criteria of Morris et $a l,{ }^{16}$ or all three, were present.

\section{Cardiac involvement}

This was established by the presence of one or more of the following: clinical symptoms, any change on colour Doppler echocardiography, electrocardiographic signs of right ventricle enlargement, pulmonary hypertension (PHT) measured by Doppler in patients with tricuspid regurgitation, ${ }^{17}$ left ventricular ejection fraction lower than $50 \%$ or right ventricular ejection fraction lower than $40 \%$ on radionuclide ventriculography, or reversible thallium perfusion defects after cold stimulation. ${ }^{18}$

\section{Renal involvement}

Renal involvement secondary to SSc was diagnosed when increased serum creatinine concentration or abnormal urine analysis (haematuria, proteinuria $>500 \mathrm{mg} / 24 \mathrm{~h}$ ) were detected in absence of any other known cause, or when a sclerodermal renal crisis defined according to Traub et $a l^{19}$ was noted.

Visceral involvement was assessed at least yearly in all patients.

Nailfold capillaroscopy was performed on each finger of both hands with a Wild M3 stereomicroscopy and Intralux 5000 Volpi. 
Table 2 Ten year survival according to age at onset, sex, and clinical variables statistically significant in patients with interval between first symptom and diagnosis equal or lower than five years

\begin{tabular}{|c|c|c|c|c|c|c|}
\hline Prognostic factors & Number & Dead & Survival ${ }^{\star}$ & $S E M$ & $\begin{array}{l}\text { Log rank } \\
\text { test }\end{array}$ & $p$ Value \\
\hline \multicolumn{7}{|l|}{ Age at onset (y) } \\
\hline$<60$ & 28 & 6 & 0.712 & 0.104 & & \\
\hline 60 & 9 & 3 & 0.583 & 0.186 & 2.87 & 0.090 \\
\hline \multicolumn{7}{|l|}{ Sex } \\
\hline Male & 5 & 1 & 0.500 & 0.354 & & \\
\hline Female & 32 & 8 & 0.687 & 0.972 & 0.01 & 0.903 \\
\hline \multicolumn{7}{|c|}{ Skin involvement groups } \\
\hline Limited & 24 & 4 & 0.750 & 0.146 & & \\
\hline Diffuse & 13 & 5 & 0.514 & 0.116 & 4.13 & 0.0421 \\
\hline \multicolumn{7}{|c|}{ FC $<70 \%$ expected value } \\
\hline Absent & 30 & 3 & 0.825 & 0.097 & & \\
\hline Present & 6 & 6 & 0.000 & 0.000 & 37.24 & $<0.001$ \\
\hline \multicolumn{7}{|l|}{ Interstitial pattern } \\
\hline Absent & 23 & 1 & 0.909 & 0.087 & & \\
\hline Present & 14 & 8 & 0.305 & 0.142 & 17.65 & $<0.001$ \\
\hline \multicolumn{7}{|l|}{ PHT } \\
\hline Absent & 32 & 4 & 0.853 & 0.069 & & \\
\hline Present & 5 & 5 & 0.000 & 0.000 & 15.33 & $<0.001$ \\
\hline \multicolumn{7}{|c|}{ PHT detected by Doppler } \\
\hline Absent & 31 & 5 & 0.797 & 0.893 & & \\
\hline Present & 4 & 3 & 0.250 & 0.216 & 4.91 & 0.026 \\
\hline \multicolumn{7}{|c|}{ Pericardial effusion } \\
\hline Absent & 24 & 2 & 0.890 & 0.741 & & \\
\hline Present & 11 & 6 & 0.254 & 0.197 & 9.21 & 0.024 \\
\hline \multicolumn{7}{|l|}{ Renal involvement } \\
\hline Absent & 31 & 3 & 0.884 & 0.063 & & \\
\hline Present & 6 & 6 & 0.000 & 0.000 & 23.03 & $<0.001$ \\
\hline
\end{tabular}

^Survival estimated according Kaplan-Meier method. FVC: forced vital capacity in pulmonary functional testing. PHT: pulmonary hypertension.

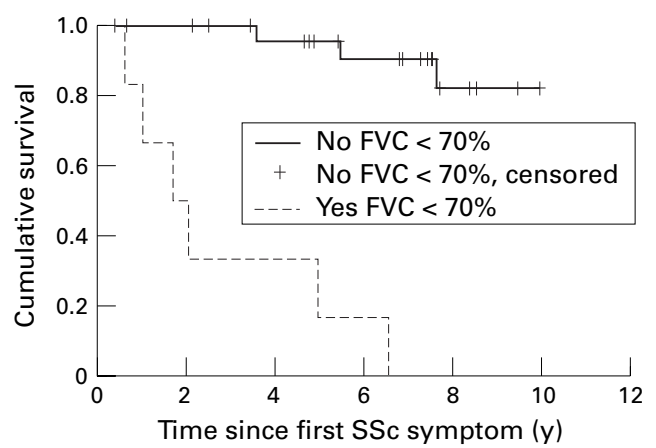

Figure 2 Survival according to forced vital capacity on pulmonary functional testing.

According to Maricq et al, ${ }^{20}$ two capillaroscopic patterns were distinguished: an active pattern characterised by predominance of capillary loss, and a slow pattern characterised by megacapillaries with no capillary loss.

LABORATORY ANALYSIS

General haematological and biochemical parameters were determined. Antinuclear antibodies were detected by immunofluorescence assay. ACA were determined by immunofluorescence technique with HEp-2 cell substract and anti-topoisomerase I antibodies were determined by immunoblotting

QUALITY OF LIFE

Patients were classified according to the maximum degree of morbidity reached; modified Knauss $^{21}$ criteria were used. Four groups were defined, and patients were assigned to group A if they had no incapacity, to group B if they had minimum activity limitation, to group $\mathrm{C}$ if they had any limitation of normal activity, and to group D if they had serious morbidity that required repeated admissions into the hospital and long periods of rest.
STATISTICAL ANALYSIS

All data collected in the study were transferred to a DBASE-IV system database. Periods between first symptom and diagnosis time, and between first symptom and end of follow up were calculated.

Cumulative survival after first symptom has been estimated according to the Kaplan-Meier method. The association between a hypothetical prognostic factor and cumulative survival after first symptom has been assessed by log rank test and, later, by stratified log rank test. To avoid an over inclusion of patients with a good prognosis, survival analysis was restricted to patients whose interval between first symptom and diagnosis had been no longer than five years. Survival among patients with skin involvement proximal to a given cut off has been compared with survival of the rest of patients.

The prevalence of patients classified in groups $\mathrm{C}$ or D of morbidity (severe morbidity) was the percentage of patients with such degrees in relation to the total number of patients. The crude association between a hypothetical prognostic factor and the prevalence of severe morbidity was assessed by the odds ratio (OR) with $95 \%$ intervals according to Cornfield method ${ }^{22}$ (or the exact method, if advised by the statistical program). The Mantel-Haenszel method $^{23}$ was used to adjust the associations detected for length of the interval between first symptom and the start of follow up. The combined study of the possible prognostic factors for severe morbidity was conducted by multiple logistic regression models; a forward stepwise strategy was used for modelling.

Calculations were done with the statistical packages SPSS $\mathrm{v}^{24}$ and EPI-INFO v 6.0..$^{25}$

\section{Results}

DESCRIPTIVE STUDY OF PATIENTS

The study included 72 patients (65 female, seven male) with a ratio F:M, 9:1. Median age at diagnosis was 54 years (range: $8-81$ ). The median of the interval between the onset of SSc (Raynaud's phenomenon or other symptom of $\mathrm{SSc}$ ) to end of follow up was 12 years (range: $0.5-60)$.

CLINICAL AND BIOLOGICAL FEATURES

Table 1 summarises the visceral involvement, capillaroscopic findings, and immunological changes of the 72 patients with SSc. Eleven (10 female, one male) of $72(15 \%)$ patients died. Deaths were caused by the following: progressive respiratory insufficiency because of lung

Table 3 Prognostic factors. Analysis after adjustment for diffuse sclerosis

\begin{tabular}{lll}
\hline Variable & $\begin{array}{l}\text { Long rank } \\
\chi^{2} \text { test }^{\star}\end{array}$ & p Value \\
\hline FVC $<70 \%$ & 20.11 & $<0.001$ \\
Interstitial pattern & 14.96 & $<0.001$ \\
Pericardial effusion & 10.72 & 0.001 \\
Pulmonary hypertension & 9.80 & 0.002 \\
Renal involvement & 22.52 & $<0.001$ \\
\hline
\end{tabular}

*After adjustment for diffuse cutaneous sclerosis ( 1 degree of freedom). 


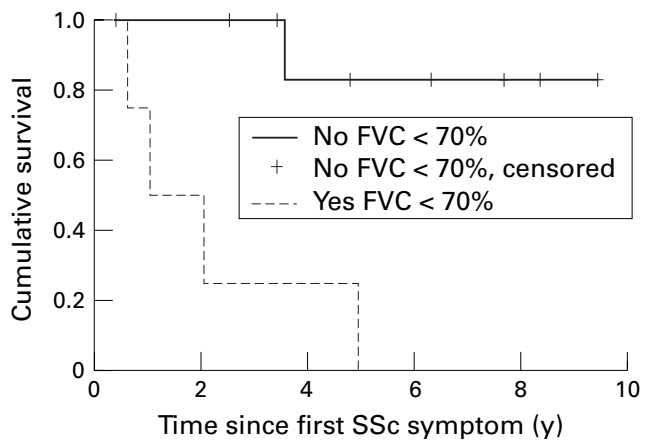

Figure 3 Survival according to forced vital capacity among patients with diffuse sclerosis. Each death is represented by a step.

fibrosis in five $(46 \%)$, three of them with secondary PHT; sclerodermal renal crisis in four $(36 \%)$ and PHT without lung fibrosis in two $(18 \%)$. According to skin groups the causes of death were distributed as follows: three with sclerodermal renal crisis and three with lung fibrosis to group 3; two with PHT and one with sclerodermal renal crisis to group 2 ; and two lung fibrosis to group 1 .

SURVIVAL

Overall survival 10 years after first SSc symptom was $85 \%$. Survival was longer for patients included in the follow up five or more years after first symptom (only two died among 35 patients); survival was $65 \%$ among the other 37 patients. To avoid an over inclusion of

Table 4 Morbidity prevalence according to clinical variables

\begin{tabular}{|c|c|c|c|c|c|}
\hline \multirow[b]{2}{*}{ Prognostic factors } & \multirow[b]{2}{*}{ Number } & \multirow{2}{*}{$\begin{array}{l}\text { Degrees of } \\
\text { morbidity }\end{array}$} & \multirow[b]{2}{*}{$\%$} & \multicolumn{2}{|l|}{ Crude } \\
\hline & & & & $O R$ & $95 \% C I$ \\
\hline \multicolumn{6}{|c|}{ Onset-diagnosis interval (y) } \\
\hline$>5$ & 35 & 9 & 25 & & \\
\hline 5 & 37 & 19 & 50 & 3.05 & $1.00,9.45$ \\
\hline \multicolumn{6}{|l|}{ Age $(y)$} \\
\hline$<60$ & 51 & 19 & 37 & & \\
\hline 60 & 21 & 9 & 43 & 0.79 & $0.25,2.54$ \\
\hline \multicolumn{6}{|l|}{ Sex } \\
\hline Male & 7 & 1 & 14 & & \\
\hline Female & 65 & 27 & 41 & 4.26 & $0.47,203.2$ \\
\hline \multicolumn{6}{|c|}{ Diffuse skin involvement } \\
\hline Absent & 56 & 13 & 23 & & \\
\hline Present & 16 & 15 & 94 & 46.57 & $6.13,2.134$ \\
\hline \multicolumn{6}{|l|}{$\mathrm{FVC}<70 \%$} \\
\hline Absent & 64 & 21 & 33 & & \\
\hline Present & 7 & 7 & 100 & $\star$ & 2.60 \\
\hline \multicolumn{6}{|l|}{ Interstitial pattern } \\
\hline Absent & 46 & 13 & 28 & & \\
\hline Present & 25 & 15 & 60 & 3.81 & $1.21,12.29$ \\
\hline \multicolumn{6}{|l|}{ PHT } \\
\hline Absent & 64 & 20 & 31 & & \\
\hline Present & 8 & 8 & 100 & $\star$ & 3.28 \\
\hline \multicolumn{6}{|c|}{ PHT (detected by Doppler) } \\
\hline Absent & 61 & 19 & 31 & & \\
\hline Present & 8 & 7 & 87 & 15.47 & $1.72,713$ \\
\hline \multicolumn{6}{|c|}{ Pericardial effusion } \\
\hline Absent & 54 & 16 & 30 & & \\
\hline Present & 15 & 10 & 66 & 4.75 & $1.21,20.23$ \\
\hline \multicolumn{6}{|c|}{ Antitopoisomerase I } \\
\hline Absent & 56 & 15 & 26 & & \\
\hline Present & 8 & 7 & 87 & 19.13 & $2.07,882.53$ \\
\hline \multicolumn{6}{|l|}{ ACA } \\
\hline Absent & 39 & 19 & 49 & & \\
\hline Present & 29 & 6 & 20 & 0.27 & $0.08,0.93$ \\
\hline \multicolumn{6}{|c|}{ Homogeneous IFI pattern } \\
\hline Absent & 57 & 18 & 31 & & \\
\hline Present & 15 & 10 & 66 & 4.24 & $1.12,18.26$ \\
\hline \multicolumn{6}{|c|}{ Active capillaroscopic } \\
\hline Absent & 46 & 8 & 18 & & \\
\hline Present & 17 & 12 & 70 & 10.82 & $2.68,51.64$ \\
\hline
\end{tabular}

p Value, Mantel-Haenszel summary $\chi^{2}$ test. ${ }^{\star}$, Uncalculable because of the absence of effectives in any cell. Abbreviations as shown in tables 2 and 3. patients with a good prognosis, survival study was restricted to the 37 patients whose interval between the first symptom and inclusion in follow up had been lower than or equal to five years.

The survival study also was made according to skin sclerosis extent and according to visceral and immunological features.

\section{Skin groups}

When survival was compared according to skin involvement cut off, significant differences were obtained only when groups 1 and 2 were combined and compared with group 3 (log rank $\chi^{2}$ test $\left.=4.13 ; 1 \mathrm{df}, \mathrm{p}=0.0421\right)$; no significant differences were observed after comparing the subgroups of patients obtained according to any other skin involvement cut off. Therefore, in subsequent analysis, patients were reclassified according to skin involvement as limited SSc defining patients with skin sclerosis distal to elbows and knees (groups 1 and 2) and diffuse SSc defining patients with skin sclerosis distal and proximal to elbows and knees (group 3). As figure 1 shows, survival was longer among patients with limited SSc.

Visceral and immunological variables

Table 2 shows the variables significantly associated with shorter survival at 10 years. Lung involvement (FVC $<70 \%$, interstitial radiological pattern, and $\mathrm{PHT}$ ) and renal involvement were associated to a poorer prognosis. As figure 2 shows, survival was shorter in patients with a FVC lower than $70 \%$ of its expected value (severe restrictive pattern).

As table 3 shows, after adjustment for diffuse cutaneous sclerosis, survival was still shorter among patients with FVC lower than $<70 \%$ of expected value, an interstitial radiological pattern, a pericardial effusion, PHT or renal involvement. Shorter survival among patients with diffuse SSc became not signicant after adjustment by severe restrictive pattern (log rank $\chi^{2}$ test $=1.02 ; 1 \mathrm{df}, \mathrm{p}=0.3116$ ).

Figure 3 shows that among patients with diffuse SSc, the four patients with FVC $<70 \%$ died within five years from first symptom, whereas only one of nine patients without severe restrictive pattern had died after this period.

MORBIDITY PREVALENCE

Table 4 shows 15 of 16 patients with diffuse sclerosis $(94 \%)$ and only 13 of 56 patients $(23 \%)$ with limited SSc were classified in degrees $\mathrm{C}$ or D of morbidity. Morbidity prevalence according to clinical variables is presented in table 4 . The variables most strongly associated with group C or D of morbidity after adjustement by the interval between the first symptom and the start of follow up were: diffuse sclerosis and PHT detected by Doppler echocardiography (table 5). When a forward stepwise strategy was used to estimate a multiple logistic regression model, only diffuse sclerosis (log likelihood ratio test $=14.23 ; 1$ df; $\mathrm{p}=0.002$ ), followed by PHT detected by Doppler echocardiography (log likelihood ratio test 
Table 5 Morbidity risk factors after adjustment by the interval between the first symptom and the diagnosis

\begin{tabular}{|c|c|c|}
\hline Prognostic factors & $O R_{M H}$ & $95 \% C I$ \\
\hline Age $<60$ years at onset & 0.67 & $0.23,1.94$ \\
\hline Female sex & 6.50 & $0.65,64.94$ \\
\hline Diffuse sclerosis & 40.92 & $4.73,353.70$ \\
\hline $\mathrm{FVC}<70 \%$ & $\star$ & \\
\hline Interstitial pattern & 3.92 & $1.35,11.40$ \\
\hline PHT & $\star$ & \\
\hline PHT by Doppler & 23.66 & $2.02,277.54$ \\
\hline Pericardial effusion & 3.73 & $1.09,12.77$ \\
\hline Homogeneous IFI pattern & 4.30 & $1.21,15.22$ \\
\hline Topoisomerase antibodies & 14.94 & $1.62,137.33$ \\
\hline $\mathrm{ACA}$ & 0.34 & $0.11,1.09$ \\
\hline Active capillaroscopic pattern & 11.74 & $2.94,46.82$ \\
\hline
\end{tabular}

$\mathrm{OR}_{\mathrm{MH}}$, OR adjusted by onset-diagnosis interval (according to Mantel-Haenszel method).

$\mathrm{p}$ Value, Mantel-Haenszel summary $\chi^{2}$ test.

$\star$, Uncalculable OR because of the absense of effectives in a cell Abbreviations as shown in tables 2 and 3 .

$=11.4 ; 1 \mathrm{df} ; \mathrm{p}=0.0007)$ were identified as independent prognostic factors. According to the final multiple logistic regression model with diffuse sclerosis and PHT, the prevalence of groups $\mathrm{C}$ or $\mathrm{D}$ of morbidity was higher among patients with diffuse sclerosis $(\mathrm{OR}=38.76 ; \mathrm{p}=$ $0.016)$ or PHT $(\mathrm{OR}=27.70 ; \mathrm{p}=0.004)$.

\section{Discussion}

There have been a number of studies examining survival after presentation with SSc, with five year survival rates varying between 34 and $73 \% .{ }^{26}$ There are a number of problems in interpreting these reports. Firstly, methodological differences between studies. Thus survival has been estimated from two different dates, both from the onset or from the diagnosis of SSc. Therefore, when authors considered survival from presentation this was subject to the problems of left censorship bias but when survival was estimated from diagnosis the interval between first symptom and diagnosis could depend more on specialist interest to SSc than on natural disease evolution. For these reasons we considered survival from disease onset defined as the self reported date of SSc first symptom. Secondly, SSc is a connective tissue disease with a variable natural history and patients could have different prognosis. Therefore it may explain survival differences found among series. We attempt to define different prognostic factors for identifying homogeneous groups of survival.

In the past decade, different classifications according to skin sclerosis extent have demonstrated their usefulness but they have not been able to identify any striking skin sclerosis cut off. $^{1-6}$ In this study, different subsets of SSc patients were compared according to a given skin sclerosis cut off. Thus, skin sclerosis extent was found a prognostic factor only when survival of SSc patients with skin sclerosis proximal to elbows and knees was compared with survival of the remaining patients. Therefore, two subsets of patients could be distinguished: limited SSc, which included patients with skin sclerosis distal to elbows and knees, and diffuse SSc formed by patients with both distal and proximal cutaneous sclerosis to elbows and knees, the last subset associated with significantly decreased survival. Interest- ingly, the survival division that we found coincides with those advocated by Medsger et al ${ }^{4}$ and LeRoy et $a \bar{l}^{6}$ but differs from the classification in three skin subsets advocated by Barnett et $a l^{1}$ and Giordano et $a l^{2}{ }^{2}$ These authors also performed longitudinal studies with SSc patients but only skin sclerosis extent was assessed as a prognostic factor. The authors obtained similar results when they compared the clinical and serological associations of extreme groups but did not when they compared intermediate forms. Thus, Barnett's type 1 was similar to Giordano's limited form and Barnett's type 3 was similar to Giordano's diffuse form. However, Barnet's type 2 and Giordano's intermediate form showed significant differences in relation to survival and serological features despite having the same skin sclerosis extent. While patients with the Giordano's intermediate form had survival and serological profiles similar to the Giordano's diffuse form, Barnett et al found significant differences between the survival of patients with type 2 and 3. Therefore, the categorisation of SSc patients into three subsets may not be accurate enough because clinical evolution and immunological findings of some patients might not be determined clearly according to these classifications.

The extent of skin sclerosis has been considered an important prognostic factor but some other authors have also considered it interesting to evaluate the prognostic value of visceral and immunological variables and they obtained different results. Farmer et al reported that generalised skin induration as well as the presence of kidney and heart involvement implied poor prognosis. Medsger et al $l^{8}$ detected more severe organic involvement in patients with the diffuse form of SSc, which was related to a bad prognosis. Bennet et al ${ }^{9}$ also observed diffuse skin sclerosis to be a prognostic factor when it was evaluated with other variables such as age, electrocardiographic findings, and renal and lung involvement. Our study enabled us to assess the prognostic value of visceral involvement as well as identifying many potentially important prognostic factors such as lung involvement (PHT and severe restrictive pattern on PFT), renal involvement or pericardial effusion. Moreover, patients with a FVC lower than $70 \%$ of its expected value had the shortest survival. Thus, among patients with diffuse SSc, the four patients affected with a severe restrictive pattern died within five years from the first symptom, whereas only one of nine patients without severe restrictive pattern had died. In contrast, diffuse sclerosis was not associated with a shorter survival after adjustment by severe restrictive pattern.

It must be remembered that SSc is a chronic disease with a high cumulative survival. As a result, patients suffering this illness may develop a severe degree of morbidity. Few studies conducted to date have evaluated morbidity prevalence in SSc patients. ${ }^{127} \mathrm{We}$ considered it of great interest to assess morbidity prevalence and identify markers of bad prognosis. In this study we found significant differences on morbidity prevalence only when 
the groups with limited SSc were combined and compared with diffuse SSc; therefore classification of SSc patients in limited and diffuse subsets was confirmed as a useful tool. More over, when clinical and immunological variables were evaluated, diffuse skin sclerosis showed the worse morbidity odds ratio followed by PHT detected by Doppler echocardiography.

On the other hand, prognostic effect of visceral involvement and skin sclerosis has been assessed recently by multivariate statistical methods $^{10-1428} 29$ but the results obtained in these studies were not applicable to the whole population of patients with SSc. Some authors ${ }^{10} 1213$ included only patients with diffuse skin sclerosis and therefore the prognostic factors were only applicable to this group of patients. Kostis et al, ${ }^{14}$ who considered skin sclerosis extent as a probable prognostic factor, observed no association between mortality and skin sclerosis extent. Bulpitt et $a l^{28}$ enrolled patients with early SSc and therefore suffering diffuse SSc because they met diagnostic criteria for SSc for less than one year; in fact, many patients with Raynaud's phenomenon for a long time before the onset of skin disease as a first manifestation of the limited SSc were excluded. Again the results obtained were not applicable to the whole group of patients with SSc. Ferri et $a l,{ }^{29}$ divided their group of SSc patients into subtypes as previously defined by Barnett et $a l^{1}$ and Giordano et $a l^{2}$ but only skin involvement and immunological changes were studied as prognostic factors. Finally, Clements et $a l,{ }^{11}$ in a study similar to ours found that skin involvement was the best prognostic factor in SSc patients. In this work when a forward stepwise strategy was used to estimate a multiple logistic regression model, only diffuse sclerosis followed by PHT detected by Doppler echocardiography were found to be independent prognostic factors.

In summary, after statistical analysis, two patient groups with different survival, morbidity, prevalence and extent of cutaneous sclerosis were established: limited SSc, with skin sclerosis distal to elbows and knees but including the face, and diffuse SSc, with skin sclerosis both distal and proximal to elbows and knees. Furthermore, lung involvement (FVC $<70 \%$ on survival study and PHT on morbidity study) was also associated with worse prognosis independently of the diffuse skin sclerosis. Despite the small number of patients included in this study we have achieved a deeper understanding of the evolution of patients with SSc.

The authors thank Christine O'Hara for helping with the English translation of this paper.

1 Barnett AJ, Miller MH, Littlejohn GO. A survival study of patients with scleroderma diagnosed over 30 years (19531983): the value of a simple cutaneous classification in the early stages of the disease. J Rheumatol 1988;15:276-83.

2 Giordano M, Valentini G, Migliaresi S, Picilo U, Vatti M. Different antibody patterns and different prognoses in patients with scleroderma with various extent of skin sclerosis. J Rheumatol 1986;13:911-6.

3 Medsger TA. Systemic sclerosis (scleroderma), eosinophilic fasciitis and calcinosis. In: McCarty DJ, ed. Arthritis and fasciitis and calcinosis. In: McCarty DJ, ed. Arthritis and
allied conditions. Philadelphia: Lea \& Febiger 1985: allied conditio
4 Medsger TA Jr. Classification of systemic sclerosis. In: Jayson MIV, Black CM, eds. Systemic sclerosis: scleroderma. London: John Wiley, 1988: 1-6.

5 LeRoy EC, Black C, Fleischmajer R, Jablonska S, Krieg T, Medsger TA, et al. Scleroderma (systemic sclerosis): classification, subsets and pathogenesis. J Rheumatol 1988;15: $202-5$.

6 LeRoy EC, Steen VD, Medsger TA Jr. Epidemiology and natural history of systemic sclerosis. Rheum Dis Clin North Am 1990;16:1-10.

7 Farmer RG, Gifford RW Jr, Hines EA Jr. Prognostic significance of Raynaud's phenomenon and other clinical characteristics of systemic scleroderma. A study of 271 cases. Circulation 1960;21:1088-95.

8 Medsger TA jr, Masi AT, Rodnan GP, Robinson H. Survival with systemic sclerosis (scleroderma). A life-table analysis of clinical and demographic factors in 309 patients. Ann Intern Med 1971;75:369-76.

9 Bennet R, Bluestone R, Holt PJL, Bywaters EGL. Survival in scleroderma. Ann Rheum Dis 1971;30:581-8.

10 Altman R, Medsger TA Jr, Bloch D, Michel B. Predictors of survival in systemic sclerosis (scleroderma). Arthritis Rheum 1991;34:403-13.

11 Clements PJ, Lachenbruch PA, Cheng S, Simmons M, Sterz M, Furst D. Skin score. A semiquantitative measure of cutaneous involvement that improves prediction of prognosis in systemic sclerosis. Arthritis Rheum 1990;33:125663.

12 Peters-Golden M, Wise R, Hochberg M, Stevens MB, Wigley FM. Carbon monoxide diffusing capacity as predictor of outcome in systemic sclerosis. Am J Med 1984;77:102734.

13 Wynn J, Fineberg N, Matzer L, Cortada X, Armstrong W, Dillon J, et al. Prediction of survival in progressive systemic sclerosis by multivariate analysis of clinical features. Am Heart J 1985;110:123-7

14 Kostis J, Seibold J, Turkevich D, Masi AT, Grau RG, Medsger TA jr, et al. Prognostic importance of cardiac arrhythmias in systemic sclerosis. Am J Med 1988;84: 1007-15.

15 Subcommittee for Scleroderma Criteria of the American Rheumatism Association Diagnostic and Therapeutic Committee: Preliminary criteria for the classification of systemic sclerosis (scleroderma). Arthritis Rheum 1980; 23:581-90.

16 Morris AH, Kanner RE, Crapo RO, Gardner RM, eds. Clinical pulmonary function testing. 2nd ed. Salt Lake City: Intermountain Thoracic Society, 1984.

17 Berger M, Haimonitz A, Tosh AV, Berdoff R, Goldberg E. Quantitative assessment of pulmonary hypertension in patients with tricuspid regurgitation using wave Doppler ultrasounds. J Am Coll Cardiol 1985;6:359.

18 Castell J, Fraile M, Candell J, Simeón CP, Fonollosa V, Alonso $\mathrm{F}$, et al. Myocardial perfusion imaging in scleroderma and Raynaud's phenomenon. Eur J Nucl Med 1992;19:660.

19 Traub YM, Shapiro AP, Rodnan GP, Medsger TA, McDonald RH, Steen VD, et al. Hypertension and renal failure (scleroderma renal crisis) in progressive systemic sclerosis. Medicine (Baltimore) 1985;62:335-52.

20 Maricq HR, Spencer-Geen G, LeRoy EC. Skin capillary abnormalities as indicators of organ involvement in scleroderma (systemic sclerosis), Raynaud's syndrome and dermatomyositis. Am J Med 1976;61:862-70.

21 Knaus WA, Zimmerman JE, Wagner DP, Draper EA, Lawrence DE. APACHE. Acute physiology and chronic health evaluation. A physiologically based classification system. Crit Care Med 1981;9: 591-7.

22 Cornfield J. A statistical problem arising from retrospective studies. In: Neyman J, ed. Proceedings of the Third Berkeley Symposium. Vol IV. Berkeley: University of California Press, 1956:135-48.

23 Mantel N, Haenszel W. Statistical aspects of the analysis of data from retrospective studies of disease. J Natl Cancer Inst 1959;22:719-48.

24 SPSS for Windows, Release 6.0. Professional statistics. Chicago: SPSS, 1993.

25 Dean AG, Dean JA, Coulombier D, Brendel KA, Smith DC, Burton AH, et al. Epi Info, Version 6: A Word-Processing, Database, and Statistics Program for Public Health on IBM-compatible Microcomputers. Atlanta: Centers for Disease Control and Prevention, 1995.

26 Silman AJ. Scleroderma and survival: A review. Ann Rheum Dis 1990;50:267-9.

27 Bole J, Steen V. The use of the Health Assessment Questionnaire (HAQ) to determine the physical disability in systemic sclerosis. Arthritis Care and Research 1991;4: $27-31$.

28 Bulpitt K, Clements P, Lachenbruch P, Paulus H, Peter J, Agopian $\mathrm{M}$, et al. Early undifferentiated connective tissue disease: III. outcome and prognostic indicators in early scleroderma (systemic sclerosis). Ann Intern Med 1993; 118:602-9.

29 Ferri C, Bernini L, Cecchetti R, Latorraga A, Marotta G, Pasero G, et al. Cutaneous and serologic subsets of systemic sclerosis. J Rheumatol 1991;18:1826-32. 